УДК 004.93’12

\title{
МОДЕЛИ И АЛГОРИТМЫ РАСПОЗНАВАНИЯ ЦИФРОВЫХ ИЗОБРАЖЕНИЙ В УСЛОВИЯХ ВОЗДЕЙСТВИЯ ДЕФОРМИРУЮЩИХ И АДДИТИВНЫХ ИСКАЖЕНИЙ
}

\author{
А. В. Акимов, А. О. Донских, А. А. Сирота \\ Воронежский государственный университет
}

Поступила в редакцию 29.01.2018 г.

\begin{abstract}
Аннотация. Рассматривается задача распознавания цифровых изображений в условиях воздействия деформирующих искажений и аддитивного шума. Синтезированы и исследованы: параметрический, на основе гауссовского приближения, непараметрический, основанный на использовании ядерных оценок функций правдоподобия, а также нейросетевой алгоритмы распознавания. Предложены модифицированные смешанные оценки функций правдоподобия, на основе свертки ядерной оценки, полученной по выборке исходных изображений, подвергнутых деформации, с плотностью распределения аддитивного шума. Теоретически и экспериментально показано, что использование смешанной оценки эквивалентно реализации процедуры искусственного размножения обучающих данных в соответствии с известной статистической моделью аддитивного шума. Проведено моделирование алгоритмов и выполнено сравнение их работы при различных значениях отношения сигнал-шум и коэффициента корреляции аддитивного шума.

Ключевые слова: распознавание цифровых изображений; деформирующие искажения; непараметрические оценки функции правдоподобия, размножение элементов обучающей выборки.

Annotation. The problem of digital image recognition under influence of warping and additive noise has been considered. Parametric, based on Gaussian approximation, and nonparametric, based on kernel density estimation of likelihood functions, along with neural network, recognition algorithms have been synthesized and analyzed. Modified mixed nonparametric estimation of likelihood functions, based on convolution of kernel estimation for original warped images data set with additive noise probability density function, has been proposed. It has been theoretically shown and experimentally proved that use of mixed nonparametric estimation equivalents to data sets augmentation according to known statistical model of additive noise. These algorithms were simulated and compared at different values of signal-to-noise ratios and additive noise correlation coefficients.
\end{abstract}

Keywords: digital image recognition, warping, nonparametric estimation of likelihood functions, data sets augmentation.

\section{ВВЕДЕНИЕ}

Во многих практически важных ситуациях в ходе цифровой обработки сигналов и изображений наряду с наличием аддитивного шума возникает специфический вид помеховых воздействий нелинейного характера - деформирующие искажения (ДИ). ДИ

(c) Акимов А. В., Донских А. О., Сирота А. А., 2018 можно воспринимать как особый вид случайных помеховых воздействий, оказывающих влияние на форму распознаваемых сигналов и изображений и имеющих высокий уровень корреляции по времени или по пространству. При обработке сигналов подобная ситуация встречается в задачах извлечения данных (data mining) [1, 2], распознавания речи [3], жестов и движений $[4,5]$, а также при анализе движущегося потока объектов, например, 
элементов зерновых смесей в системах сепарации реального времени [6].

Применительно к обработке изображений влияние ДИ соответствуют естественной изменчивости содержимого сцены при нелинейном относительно друг друга перемещении отдельных ее элементов $[7,8]$. Такие ситуации возникают, например, при выражении различных эмоций на лице человека, смене ракурса при съемке или в условиях наличия аберраций оптической системы [9], используемой при регистрации изображений. Кроме того, как деформирующие искажения можно трактовать естественные изменения формы одного класса объектов, варьирующихся от экземпляра к экземпляру (например, в биологии - медицине [10] или ботанике [6]).

В рамках этого направления подавляющее большинство предшествующих работ посвящено анализу влияния деформирующих искажений при решении разнообразных практических задач:

- сопоставление временных рядов для разного рода сигналов [1-6];

- исправление возникающих при съемке оптических искажений [9];

- расчет оптического потока при последовательной регистрации изображений в процессе изменения положений и ориентации объектов сцены в разные моменты времени, например, при их перемещениях или при смене ракурса съемки [11, 12];

- выполнение «морфинга» (morphing) процесса постепенного замещения одного изображения другим путем плавных изменений интенсивностей пикселей, положений и формы схожих в некоем смысле частей этих изображений $[13,14]$;

- при учете различий в форме анализируемых объектов в разнообразных моделях распознавания образов $[7,8,10]$, в том числе при искусственном размножении обучающих выборок во время построения современных алгоритмов машинного обучения $[15,16]$.

Действительно, использование моделей деформирующих искажений является важным для построения решающих правил, основанных на концепциях машинного обучения. Как известно, поиск и подготовка обучающих данных к использованию часто требуют значительных затрат времени и ресурсов. В ряде случаев сбор необходимого числа образов оказывается сложен или даже невозможен из-за специфического характера предметной области. В этом плане с учетом необходимости обеспечения представительности обучающих данных одним из возможных подходов представляется искусственное размножение ограниченного набора «опорных» образов на основе стохастических или детерминистских моделей преобразования, реализующих необходимые деформации. В подобной постановке ДИ могут являться существенным инструментом формирования обучающей выборки, содержащей множество образов анализируемых объектов $[15,16]$.

Тем не менее, строгого решения задача синтеза и анализа алгоритмов распознавания изображений с учетом влияния деформирующих искажений в известных источниках [1-16] не получила: предложенные в них алгоритмические подходы носят преимущественно эвристический характер. В связи с этим представляет интерес постановка и решение задачи распознавания объектов, представленных цифровыми сигналами и изображениями случайной формы в условиях деформирующих искажений, как в рамках статистической теории решений (исследование для сигналов проведено авторами в [17]), так и на базе методов машинного обучения, и получение на этой основе оптимальных и квазиоптимальных алгоритмов обработки информации. Таким образом, целью работы является проведение синтеза и сравнительного анализа алгоритмов распознавания цифровых изображений на основе моделей, учитывающих воздействие деформирующих искажений и аддитивных помех.

\section{2. МЕТОДЫ И МАТЕРИАЛЫ}

В качестве базового метода исследования в работе используется метод статистического синтеза алгоритмов распознавания на основе задания модели воздействующих искажений и помех, а также на основе непараметрических ядерных оценок многомер- 


\section{А. В. Акимов, А. О. Донских, А. А. Сирота}

ных распределений используемых признаков распознавания. Как альтернативный вариант рассматриваются возможности построения алгоритмов машинного обучения на базе размножения обучающих данных с привлечением для этой цели непараметрических ядерных оценок. При проведении исследований алгоритмов использовались как искусственно синтезированные изображения, так и реальные изображения биологических объектов применительно к задаче анализа неоднородного потока зерновых смесей в системах сепарации реального времени [6].

2.1. Исходная статистическая модель и оптимальный алгоритм распознавания. Пусть без существенного ограничения общности на множестве значений $\Omega_{x y}=\Omega_{x} \times \Omega_{y}$, $\Omega_{x}=\left[0, x_{\max }\right], \Omega_{y}=\left[0, y_{\max }\right]$ заданы изображения известной формы, определяющие эталонные описания двух классов образов $\omega_{1}: S_{1}(x, y), \omega_{2}: S_{2}(x, y),(x, y) \in \Omega_{x y}$. Наблюдению доступны реализации случайных функций, формируемые на основе этих изображений при воздействии ДИ и аддитивного гауссовского шума,

$$
\mathrm{U}_{i}(x, y)=\tilde{\mathrm{S}}_{i}(x, y)+\mathrm{V}(x, y)=
$$

$=\mathrm{S}_{i}\left[x+\mathrm{A}_{x}(x, y), y+\mathrm{A}_{y}(x, y)\right]+\mathrm{V}(x, y), i=\overline{1,2}$, где $\mathrm{U}_{i}(x, y)$ - реализация случайной функции, описывающей наблюдаемое изображение; $\tilde{S}_{i}(x, y)$ - реализация случайной функции, описывающей деформированное изображение; $\mathrm{A}(x, y)=\left(\mathrm{A}_{x}(x, y), \mathrm{A}_{y}(x, y)\right)^{\mathrm{T}}$ - однозначная дифференцируемая вектор-функция координат, являющаяся реализацией векторного случайного поля, описывающего ДИ вдоль осей изображений $x$ и $y$ соответственно, такая, что $\left(x+\mathrm{A}_{x}(x, y), y+\mathrm{A}_{y}(x, y)\right)^{\mathrm{T}} \in \Omega_{x y} ; \mathrm{V}(x, y)-$ реализация скалярного случайного поля, описывающего действие аддитивного гауссовского шума с параметрами $\mathrm{M}[\mathrm{V}(x, y)]=0$, $\mathrm{M}\left[\mathrm{V}(x, y) \mathrm{V}\left(x^{\prime}, y^{\prime}\right)^{\mathrm{T}}\right]=\sigma^{2} \mathrm{R}\left(x-x^{\prime}, y-y^{\prime}\right)$.

Векторное случайное поле $\mathrm{A}(x, y)=$ $=\left(\mathrm{A}_{x}(x, y), \mathrm{A}_{y}(x, y)\right)^{\mathrm{T}}$ в общем случае может быть представлено своей плотностью распределения, а в простейшем случае математическим ожиданием $\mathrm{m}(x, y)=M[\mathrm{~A}(x, y)],(x, y) \in \Omega_{x y}$ и функцией корреляции, $\mathrm{B}\left(x, x^{\prime}, y, y^{\prime}\right)=$ $=M\left[(\mathrm{~A}(x, y)-\mathrm{m}(x, y))\left(\mathrm{A}\left(x^{\prime}, y^{\prime}\right)-\mathrm{m}\left(x^{\prime}, y^{\prime}\right)\right)^{\mathrm{T}}\right]$.
Без существенного ограничения общности предположим, что форма функции ДИ по каждой координате определяется реализациями случайных полей $\mathrm{A}_{x}(x, y), \mathrm{A}_{y}(x, y)$. Здесь можно рассмотреть две ситуации: случайные поля $\mathrm{A}_{x}(x, y), \mathrm{A}_{y}(x, y)$ независимы и, наоборот, полностью зависимы, т. е. фактическое воздействие осуществляется общим случайным полем $\mathrm{A}_{x y}(x, y)=\mathrm{A}_{x}(x, y)=\mathrm{A}_{y}(x, y)$ по каждой координате. Далее будем рассматривать первый вариант (независимая деформация по каждой координате). Реализации $\mathrm{A}_{x(y)}(x, y)$ будем задавать как произведения реализаций однородного случайного поля $\mathrm{A}_{0 x(y)}(x, y)$ с заданной функцией корреляции $\mathrm{B}_{0}\left(x-x^{\prime}, y-y^{\prime}\right) \quad$ на оконную функцию $\mathrm{O}_{x(y)}(x(y))$, используемую для ограничения значений функции деформации,

$$
\begin{gathered}
\mathrm{A}(x, y)=\left(\mathrm{O}_{x}(x) \mathrm{A}_{0 x}(x, y), \mathrm{O}_{y}(y) \mathrm{A}_{0 y}(x, y)\right)^{\mathrm{T}}, \\
\quad \mathrm{O}_{x(y)}(x(y))= \\
=\left\{\begin{array}{lc}
\frac{x(y)}{A_{m}}, & 0 \leq x(y)<A_{m}, \\
1, & A_{m} \leq x(y) \leq x(y)_{\max }-A_{m}, \\
\frac{x(y)_{\max }-x(y)}{A_{m}}, x(y)_{\max }-A_{m}<x(y) \leq x(y)_{\max } .
\end{array}\right.
\end{gathered}
$$

где $A_{m}^{2}$ - дисперсия случайного поля $\mathrm{A}_{0}(x, y)$.

При цифровой обработке в результате дискретизации с интервалами $\Delta x$ и $\Delta y$ формируются цифровые эквиваленты наблюдаемых в непрерывном времени изображений путем фиксации их значений по координатам $x_{k}=(k-1) \Delta x, \quad k=\overline{1, m_{x}}, \quad \Delta x=x_{\max } /\left(m_{x}-1\right)$, $x_{1}=0, \quad x_{m_{x}}=x_{\max }$ и $y_{p}=(p-1) \Delta y, \quad p=\overline{1, n_{y}}$, $\Delta y=y_{\max } /\left(n_{y}-1\right), \quad y_{1}=0, \quad y_{n_{y}}=y_{\max }$ :

$$
\begin{gathered}
u_{i}[k, p]=\mathrm{U}_{i}\left(x_{k}, y_{p}\right)= \\
=\tilde{\mathrm{S}}_{i}\left(x_{k}, y_{p}\right)+\mathrm{V}\left(x_{k}, y_{p}\right)= \\
=\mathrm{S}_{i}\left[x_{k}+\mathrm{A}_{x}\left(x_{k}, y_{p}\right), y_{p}+\mathrm{A}_{y}\left(x_{k}, y_{p}\right)\right]+ \\
+\mathrm{V}\left(x_{k}, y_{p}\right), i=\overline{1,2},
\end{gathered}
$$

заданные на двумерной дискретной сетке $\Omega_{k p}=\left\{k=\overline{1, m_{x}}, p=\overline{1, n_{y}}\right\}$. В качестве эквивалентного описания этих изображений и случайных полей будем использовать их представление в виде векторов

$\mathbf{u}_{1} \in \mathbf{R}^{n}, \mathbf{u}_{2} \in \mathbf{R}^{n}, \mathbf{s}_{1} \in \mathbf{R}^{n}, \mathbf{s}_{2} \in \mathbf{R}^{n}, \tilde{\mathbf{s}}_{1} \in \mathbf{R}^{n}, \tilde{\mathbf{s}}_{2} \in \mathbf{R}^{n}$, 
Модели и алгоритмы распознавания цифровых изображений ...

$$
\mathbf{a} \in \mathbf{R}^{n}, \mathbf{v} \in \mathbf{R}^{n}, n=m_{x} \times n_{y},
$$

полученных в ходе развертки исходных функций по столбцам. Для функции внесения ДИ: $\mathrm{A}(x, y)=\left(\mathrm{A}_{x}(x, y), \mathrm{A}_{y}(x, y)\right)^{\mathrm{T}}, \quad$ соответствующий развернутый вектор а определяется соотношением: $\tilde{s}_{i}\left[x_{k}\right]=s_{i}\left[x_{k}+a_{k}\right]$.

Пусть исходные изображения появляются на входе распознающей системы с априорными вероятностями $p\left(\omega_{i}\right), i=\overline{1,2}$ в виде вектора наблюдений $\mathbf{x}=\left(x_{1}, \ldots, x_{n}\right)^{\mathrm{T}}$, принадлежащего к одному из двух классов $\omega_{1}: \mathbf{x}=\mathbf{u}_{1}, \omega_{2}: \mathbf{x}=\mathbf{u}_{2}$. Требуется сформировать решение, относящее каждое наблюдение к тому или иному классу. Как известно $[18,19]$, в этом случае решение задачи при использовании простой функции потерь определяется оптимальным решающим правилом вида

$$
\mathrm{l}(x)=\frac{p\left(\mathbf{x} / \omega_{1}\right)}{p\left(\mathbf{x} / \omega_{2}\right)}{ }_{\omega_{2}}^{>} l_{0}=\frac{p\left(\omega_{2}\right)}{p\left(\omega_{1}\right)},
$$

где $\mathrm{l}(x)$ - величина отношения функций правдоподобия (ФП) классов, сравниваемая при принятии решения с порогом $l_{0}$. Далее будем считать априорные вероятности одинаковыми $\left(l_{0}=1\right)$.

Ранее, в [17] авторами был проведен синтез и анализ оптимальных и квазиоптимальных алгоритмов распознавания сигналов в условиях ДИ. Использованная при этом модель была основана на введении многочисленных допущений. В итоге они позволили представить внесение деформации в виде нелинейного оператора перестановки (размещения с повторениями) элементов исходного дискретного сигнала с добавлением аддитивной помеховой составляющей, вызванной ошибками квантования непрерывной функции деформации. Установлено, что применение оптимального алгоритма, основанного на вычислении ФП классов по аналогии с (1), в этом случае сводится к взвешенному суммированию условных плотностей распределения для всех возможных вариантов размещений элементов исходных сигналов, приводящих к появлению данного деформированного сигнала. При этом для ДИ, являющихся реализациями случайных функций, число возможных размещений с повторениями для сигнала из $n$ элементов составляет величину порядка $(2 m+1) ! !(2 m-1) ! ! \leq n !$, если $n=2 m+1$ нечетное, и $(2 m-1) ! !(2 m-1) ! ! \leq n !$, если $n=2 m$ четное, [17]. Перебор такого количества комбинаций, сопровождаемый соответствующими вычислениями, затрудняет практическое применение оптимального алгоритма.

Еще в большей степени эта ситуация усугубляется в задачах обработки изображений, где в случае независимости случайных полей $\mathrm{A}_{x}(x, y), \mathrm{A}_{y}(x, y)$ количество комбинаций перестановок элементов изображения составляет величину равную произведению числа возможных размещений по каждой его оси. В таких условиях работа оптимального алгоритма связана с необходимостью проведения значительных по объему вычислений, что делает его применение для обработки изображений практически невозможным. Кроме того, синтезируемые на этой основе алгоритмы оказываются чувствительными по отношению к адекватности используемых моделей данных, которые основаны на введении многочисленных допущений и приближений [17]. Поэтому, в качестве альтернативного подхода к решению задачи, целесообразно перейти к применению непараметрических ядерных оценок для восстановления ФП классов или к применению методов машинного обучения на основе использования искусственно сгенерированной выборки обучающих данных, полученной путем внесения ДИ в изображения в соответствии с используемой статистической моделью [15].

2.2. Использование непараметрических смешанных ядерных оценок. Для получения оценок ФП (1) без введения каких-либо ограничений, допущений и приближений можно воспользоваться методами непараметрического оценивания многомерных плотностей распределения вероятностей классов путем генерации обучающего множества данных по каждому из них. Решение задачи подобным образом имеет смысл для случая воздействия ДИ как реализаций случайного поля с известными статистическими характеристиками.

Пусть для проведения оценок плотностей распределения вероятностей $p\left(\mathbf{x} / \omega_{i}\right), i=\overline{1,2}$ по каждому из классов заданы обучающие 


\section{А. В. Акимов, А. О. Донских, А. А. Сирота}

данные, полученные при внесении ДИ как реализации случайного поля с заданными характеристиками,

$$
\begin{gathered}
U^{H_{i}}=\left\{\mathbf{u}_{i}^{(1)}, \ldots, \mathbf{u}_{i}^{\left(H_{i}\right)}\right\}, \mathbf{u}_{i}^{(h)}=\left(u_{i, 1}^{(h)}, \ldots, u_{i, n}^{(h)}\right)^{\mathrm{T}} \in \mathbf{R}^{n}, \\
i=\overline{1,2}, U^{H}=\bigcup_{i=1}^{2} U^{H_{i}},
\end{gathered}
$$

где $U^{H}$ - общая обучающая выборка; $H$ число ее элементов (верхний индекс в скобках соответствует элементу обучающей выборки); $n$ - размерность вектора наблюдений.

Для синтеза алгоритмов распознавания вместо плотностей $p\left(\mathbf{u} / \omega_{i}\right)$ в этом случае могут быть использованы их непараметрические оценки $\tilde{p}\left(\mathbf{u} / \omega_{i}\right)$, обладающие, желательно, свойствами несмещенности и сходимости по вероятности. Наиболее приемлемые для решаемой задачи оценки, как показывает анализ [19], могут быть получены на основе метода Парзена (ядерные оценки).

Для их использования первоначально необходимо выполнить снижение размерности вектора признаков, для которого будут строиться оценки ФП классов. Данный процесс целесообразно осуществить на основе метода главных компонент [19]. Тогда для снижения размерности используется линейное преобразование

$$
\mathbf{x}=\left(x_{1}, \ldots, x_{K}\right)^{\mathrm{T}}=\mathbf{Q}_{K}^{\mathrm{T}} \mathbf{u},
$$

где $\mathbf{Q}_{K}^{\mathrm{T}}=\left(q_{1}, \ldots, q_{K}\right)^{\mathrm{T}}-$ прямоугольная матрица размера $K \times n$ усеченного преобразования Карунена-Лоэва, строками которой являются собственные векторы выборочной матрицы ковариации исходной выборки $q_{1}, \ldots, q_{K}$, соответствующие $K$ ее максимальным собственным значениям [19]. Таким образом, при использовании оценок на основе метода Парзена далее будем рассматривать преобразованный вектор признаков $\mathbf{x}$ из (2) и соответствующие ему обучающие выборки $X^{H_{i}}=\left\{\mathbf{x}^{(i, 1)}, \ldots, \mathbf{x}^{\left(i, H_{i}\right)}\right\}$, $i=\overline{1,2}$.

Для каждого класса в отдельности производится восстановление плотности в виде

$$
\tilde{p}\left(\mathbf{x} / \omega_{i}\right)=\frac{1}{H_{i} h^{K}} \sum_{k=1}^{H_{i}} \phi\left(\frac{\mathbf{x}-\mathbf{x}^{(i, k)}}{h}\right), i=\overline{1,2},
$$

где $h^{-K} \phi\left(\left\{\mathbf{x}-\mathbf{x}^{(i, k)}\right\} / h\right)$ - функция ядра, центрированная относительно каждого вектора обучающей выборки класса; $h$ - параметр оконной функции. Полученные таким обра- зом оценки затем используются при построении алгоритмов принятия решения на основе (1) в виде

$$
\frac{H_{1}}{H_{1}+H_{2}} \tilde{p}\left(\mathbf{x} / \omega_{1}\right)_{<}^{>} \frac{H_{2}}{H_{1}+H_{2}} \tilde{p}\left(\mathbf{x} / \omega_{2}\right) .
$$

$\omega_{2}$

В качестве универсальной функции ядра, подходящей для многих случаев, целесообразно использовать функцию вида [19]:

$$
\begin{gathered}
\frac{1}{h^{K}} \phi\left(\frac{\mathbf{x}-\mathbf{x}^{(i, k)}}{h}\right)= \\
=\frac{1}{(2 \pi)^{K / 2} h^{K}\left|\boldsymbol{\Xi}_{i}\right|^{1 / 2}} \times \\
\times \exp \left(-\frac{\left(\mathbf{x}-\mathbf{x}^{(i, k)}\right)^{\mathrm{T}} \boldsymbol{\Xi}_{i}^{-1}\left(\mathbf{x}-\mathbf{x}^{(i, k)}\right)}{2 h^{2}}\right)= \\
=N\left(\mathbf{x}, \mathbf{x}^{(i, k)}, h^{2} \boldsymbol{\Xi}_{i}\right),
\end{gathered}
$$

где $N\left(\mathbf{x}, \mathbf{x}^{(i, k)}, h^{2} \boldsymbol{\Xi}_{i}\right)$ - обозначение гауссовской плотности распределения с математическим ожиданием $\mathbf{X}^{(i, k)}$ и матрицей ковариаций $h^{2} \boldsymbol{\Xi}_{i}$, где $\boldsymbol{\Xi}_{i}$ задается исходя из выборочных оценок матриц ковариаций классов $\boldsymbol{\Xi}_{i}=\tilde{\mathbf{C}}_{i}, \quad i=\overline{1,2}$ или в виде диагональной матрицы $\boldsymbol{\Xi}_{i}=\mathbf{I}$.

Для выбора $h\left(H_{i}\right)$ в [18] рекомендуется использовать соотношение $h\left(H_{i}\right)=H_{i}^{-s / K}$, $0<s<0,5$. При большой размерности вектора признаков используются более сложные методики $[19,20]$, среди которых наибольшее распространение получила методика максимизации функционала правдоподобия при реализации метода скользящего контроля (кросс-валидации). При наличии существенной неравномерности распределений данных величина этого параметра может задаваться переменной на основе анализа расстояний до $k$-ближайших соседей $[18,21]$.

В интересах решения рассматриваемой задачи распознавания изображений в условиях наличия как деформирующих, так и аддитивных помех предлагается модификация алгоритма построения ядерных оценок для случая воздействия аддитивного шума с известной плотностью распределения $p(\mathbf{v})$. Она заключается в формировании статистических описаний классов в два этапа.

1. На первом этапе на основе генерации множества деформаций изображений в от- 
сутствии аддитивного шума формируются выборки $\breve{X}^{H_{i}}=\left\{\breve{\mathbf{x}}^{(i, 1)}, \ldots, \breve{\mathbf{x}}^{\left(i, H_{i}\right)}\right\}, \quad i=1,2$, на основе которых строятся ядерные оценки плотностей $\tilde{p}\left(\widetilde{\mathbf{x}} / \omega_{i}\right)$, где $\breve{\mathbf{x}}=\mathbf{x}-\mathbf{v}$.

2. На втором этапе осуществляется свертка полученной оценки распределения вектора признаков $\tilde{p}\left(\widetilde{\mathbf{x}} / \omega_{i}\right)$ с плотностью распределения аддитивного шума в соответствии с соотношением:

$$
\hat{p}\left(\mathbf{x} / \omega_{i}\right)=\int \tilde{p}\left(\mathbf{x}-\mathbf{v} / \omega_{i}\right) p(\mathbf{v}) d \mathbf{v} .
$$

Рассмотрим пример использования ядра вида (3) для непараметрических оценок в присутствии аддитивного гауссовского шума, описываемого распределением $p(\mathbf{v})=N\left(\mathbf{v}, 0, \mathbf{C}_{\mathbf{v}}\right)$. чем

Пусть $\mathbf{x}$ и $\mathbf{z}$ два гауссовских вектора, при$p_{\mathbf{x}}(\mathbf{x})=N\left(\mathbf{x}, \mathbf{m}_{\mathbf{x}}, \mathbf{C}_{\mathbf{x}}\right) ; p_{\mathbf{z}}(\mathbf{z} / \mathbf{x})=N\left(\mathbf{z}, \mathbf{H x}, \mathbf{C}_{\mathbf{z}}\right)$, где $\mathbf{H}$ - матрица соответствующей размерности. Тогда плотность распределения вектора $\mathbf{z}$ имеет вид [22]

$$
\begin{aligned}
p_{\mathbf{z}}(\mathbf{z}) & =\int N\left(\mathbf{z}, \mathbf{H x}, \mathbf{C}_{\mathbf{z}}\right) N\left(\mathbf{x}, \mathbf{m}_{\mathbf{x}}, \mathbf{C}_{\mathbf{x}}\right) d \mathbf{x}= \\
& =N\left(\mathbf{z}, \mathbf{H} \mathbf{m}_{\mathbf{x}}, \mathbf{H C}_{\mathbf{x}} \mathbf{H}^{\mathrm{T}}+\mathbf{C}_{\mathbf{z}}\right) .
\end{aligned}
$$

Отсюда при использовании гауссовского ядра (3) и гауссовской плотности аддитивного шума получается оценка в виде

$$
\hat{p}\left(\mathbf{x} / \omega_{i}\right)=\frac{1}{H_{i}} \sum_{k=1}^{H_{i}} N\left(\mathbf{x}, \breve{\mathbf{x}}^{(i, k)}, h^{2} \boldsymbol{\Xi}_{i}+\mathbf{C}_{\mathbf{v}}\right) .
$$

Полученные таким образом оценки плотностей распределения будем назвать смешанными, акцентируя внимание на том, что одна из составляющих смеси (4) получена на основе непараметрической оценки по обучающей выборке, а другая изначально задана в аналитическом виде.

\section{3. Анализ свойств смешанных оценок.} Как известно $[18,21]$, непосредственные оценки Парзена при определенных условиях являются несмещенными и состоятельными. Пусть для оценки $\tilde{p}\left(\mathbf{x} / \omega_{i}\right)$ выполняются следующие свойства функции ядра:

$$
\begin{gathered}
\frac{1}{h^{K}} \phi\left(\frac{\mathbf{x}-\mathbf{x}^{(i, k)}}{h}\right)=\frac{1}{h^{K}} \phi(\mathbf{u} / h), \int \frac{1}{h^{K}} \phi(\mathbf{u} / h) d \mathbf{u}=1, \\
\int \frac{1}{h^{K}}|\phi(\mathbf{u} / h)| d \mathbf{u}<\infty, \sup |\phi(\mathbf{u} / h)|<\infty,
\end{gathered}
$$

что говорит о том, что ядро удовлетворяет свойствам плотности распределения [19].
Пусть также при задании параметра ядра $h$ как функции наблюдений $h\left(H_{i}\right)$, где $h^{k}\left(H_{i}\right) \underset{H_{i} \rightarrow \infty}{\rightarrow} 0, \quad H_{i} h^{k}\left(H_{i}\right) \underset{H_{i} \rightarrow \infty}{\rightarrow} \infty \quad$ [19] и $\psi_{H_{i}}(\mathbf{u})=h^{-K}\left(H_{i}\right) \phi\left(\mathbf{u} / h\left(H_{i}\right)\right)$, для каждой точки непрерывности исходной плотности $p\left(\mathbf{x} / \omega_{i}\right)$ выполняется

$$
\begin{gathered}
\int \psi_{H_{i}}(\mathbf{x}-\mathbf{s}) p\left(\mathbf{s} / \omega_{i}\right) d \mathbf{s} \underset{H_{i} \rightarrow \infty}{\rightarrow} p\left(\mathbf{x} / \omega_{i}\right), \\
\frac{1}{H_{i}} \sup \psi_{H_{i}}(\mathbf{x}) \underset{H_{i} \rightarrow \infty}{\rightarrow} 0 .
\end{gathered}
$$

Тогда оценка $\tilde{p}\left(\mathbf{x} / \omega_{i}\right)$ является состоятельной в среднем квадратичном в каждой точке непрерывности $p\left(\mathbf{x} / \omega_{i}\right)$ [23]. Отметим, что первое из этих условий означает, что $\psi_{H_{i}}(\mathbf{x}-\mathbf{s})$ при $H_{i} \rightarrow \infty$ сходится к дельта-функции с центром в точке $\mathbf{s}$. Для широкого класса функций, которые могут быть использованы в качестве ядра и обладают свойствами (6), такая сходимость имеет место, если $h^{k}\left(H_{i}\right) \underset{H_{i} \rightarrow \infty}{\rightarrow} 0$. Второе условие для таких функций выполняется, если $H_{i} h^{k}\left(H_{i}\right) \underset{H_{i} \rightarrow \infty}{\rightarrow} \infty$ [23].

При этом асимптотическая несмещенность оценки $\tilde{p}\left(\mathbf{x} / \omega_{i}\right)$, полученной по совокупности обучающей выборки $X^{H_{i}}=\left\{\mathbf{x}^{(i, 1)}, \ldots, \mathbf{x}^{\left(i, H_{i}\right)}\right\}$, может быть показана на основе следующих преобразований [23]:

$$
\begin{gathered}
M\left[\tilde{p}\left(\mathbf{x} / \omega_{i}\right)\right]= \\
=\frac{1}{H_{i} h^{K}} \sum_{k=1}^{H_{i}} M\left[\phi\left(\frac{\mathbf{x}-\mathbf{x}^{(i, k)}}{h}\right)\right]= \\
=\frac{1}{h^{K}} M\left[\phi\left(\frac{\mathbf{x}-X^{H_{i}}}{h}\right)\right]= \\
=\int \frac{1}{h^{K}} \phi\left(\frac{\mathbf{x}-\mathbf{s}}{h}\right) p\left(\mathbf{s} / \omega_{i}\right) d \mathbf{s}= \\
\int \psi_{H_{i}}(\mathbf{x}-\mathbf{s}) p\left(\mathbf{s} / \omega_{i}\right) d \mathbf{s} \underset{H_{i} \rightarrow \infty}{\rightarrow} p\left(\mathbf{x} / \omega_{i}\right) .
\end{gathered}
$$

Утверждение 1. Если плотность распределения $\tilde{p}\left(\mathbf{x} / \omega_{i}\right)$ при $h^{k}\left(H_{i}\right) \underset{H_{i} \rightarrow \infty}{\rightarrow} 0$ и $H_{i} h^{k}\left(H_{i}\right) \underset{H_{i} \rightarrow \infty}{\rightarrow} \infty$ является асимптотически несмещенной оценкой плотности $p\left(\mathbf{x} / \omega_{i}\right)$ для всех $\mathbf{x}$, то и смешанная оценка $\hat{p}\left(\mathbf{x} / \omega_{i}\right)$ является асимптотически несмещенной оценкой $p\left(\mathbf{x} / \omega_{i}\right)$.

Пусть $X^{H_{i}}=\left\{\mathbf{x}^{(i, 1)}, \ldots, \mathbf{x}^{\left(i, H_{i}\right)}\right\}$, где каждый $\mathbf{x}^{(k)}=\breve{\mathbf{x}}^{(k)}+\mathbf{v}^{(k)}, \quad \breve{X}^{H_{i}}=\left\{\breve{\mathbf{x}}^{(i, 1)}, \ldots, \breve{\mathbf{x}}^{\left(i, H_{i}\right)}\right\}$, $V=\left\{\mathbf{v}^{(1)}, \ldots, \mathbf{v}^{\left(H_{i}\right)}\right\}$. Как показано выше, для стандартной оценки $\tilde{p}\left(\mathbf{x} / \omega_{i}\right)$ выполняется (7). С другой стороны 


$$
\begin{gathered}
M\left[\tilde{p}\left(\mathbf{x} / \omega_{i}\right)\right]= \\
=\frac{1}{H_{i} h^{K}} \sum_{k=1}^{H_{i}} M\left[\phi\left(\frac{\mathbf{x}-\breve{\mathbf{x}}^{(i, k)}-\mathbf{v}^{(k)}}{h}\right)\right]= \\
=\frac{1}{h^{K}} M\left[\phi\left(\frac{\mathbf{x}-\breve{X}^{H_{i}}-V}{h}\right)\right]= \\
=\int \frac{1}{h^{K}} \phi\left(\frac{\mathbf{x}-\breve{\mathbf{s}}-\mathbf{v}}{h}\right) p\left(\breve{\mathbf{s}} / \omega_{i}\right) p(\mathbf{v}) d \breve{\mathbf{s}} d \mathbf{v}= \\
=\int \vartheta_{H_{i}}(\mathbf{x}-\breve{\mathbf{s}}) p\left(\breve{\mathbf{s}} / \omega_{i}\right) d \breve{\mathbf{s}}= \\
=M\left[\vartheta_{H_{i}}\left(\mathbf{x}-\breve{X}^{H_{i}}\right)\right]=M\left[\hat{p}\left(\mathbf{x} / \omega_{i}\right)\right] .
\end{gathered}
$$

Запись $\int \vartheta_{H_{i}}(\mathbf{x}-\breve{\mathbf{s}}) p\left(\breve{\mathbf{s}} / \omega_{i}\right) d \breve{\mathbf{s}}$ использует функцию, суммируемую в представлении смешанной оценки (4), и, следовательно, стандартная оценка имеет то же математическое ожидание, что и смешанная, т. е. для нее также выполняется свойство асимптотической несмещенности.

Как известно, если оценка сходится к истинному значению параметра «в среднем квадратичном» или если оценка является асимптотически несмещенной при стремлении ее дисперсии к нулю, то такая оценка будет состоятельной. Таким образом, состоятельность стандартной оценки $\tilde{p}\left(\mathbf{x} / \omega_{i}\right)$ вытекает из ее сходимости в среднеквадратичном, что может быть показано на основе следующих преобразований $[18,23]$ :

$$
\begin{gathered}
D\left[\tilde{p}\left(\mathbf{x} / \omega_{i}\right)\right]=M\left[\tilde{p}^{2}\left(\mathbf{x} / \omega_{i}\right)\right]-p^{2}\left(\mathbf{x} / \omega_{i}\right), \\
M\left[\tilde{p}^{2}\left(\mathbf{x} / \omega_{i}\right)\right]= \\
=\frac{1}{H_{i}^{2} h^{2 K}} \sum_{r=1}^{H_{i}} \sum_{s=1}^{H_{i}} M\left[\phi\left(\frac{\mathbf{x}-\mathbf{x}^{(i, r)}}{h}\right) \phi\left(\frac{\mathbf{x}-\mathbf{x}^{(i, s)}}{h}\right)\right]= \\
=\frac{1}{H_{i}^{2} h^{2 K}} \sum_{r=1}^{H_{i}} M\left[\phi^{2}\left(\frac{\mathbf{x}-\mathbf{x}^{(i, r)}}{h}\right)\right]+ \\
+\frac{1}{H_{i}^{2} h^{2 K}} \sum_{r=1}^{H_{i}} \sum_{s=1, s \neq r}^{H_{i}} M\left[\phi\left(\frac{\mathbf{x}-\mathbf{x}^{(i, r)}}{h}\right) \phi\left(\frac{\mathbf{x}-\mathbf{x}^{(i, s)}}{h}\right)\right]= \\
=\frac{1}{H_{i}} \int \frac{1}{h^{2 K}} \phi^{2}\left(\frac{\mathbf{x}-\mathbf{s}}{h}\right) p\left(\mathbf{s} / \omega_{i}\right) d \mathbf{s}+ \\
+\frac{H_{i}-1}{H_{i}}\left(\int \frac{1}{h^{K}} \phi\left(\frac{\mathbf{x}-\mathbf{s}}{h}\right) p\left(\mathbf{s} / \omega_{i}\right) d \mathbf{s}\right)^{2}= \\
=\frac{1}{H_{i}} \int \psi_{H_{i}}^{2}(\mathbf{x}-\mathbf{s}) p\left(\mathbf{s} / \omega_{i}\right) d \mathbf{s}+
\end{gathered}
$$

$$
+\frac{H_{i}-1}{H_{i}}\left(\int \psi_{H_{i}}(\mathbf{x}-\mathbf{s}) p\left(\mathbf{s} / \omega_{i}\right) d \mathbf{s}\right)^{2} .
$$

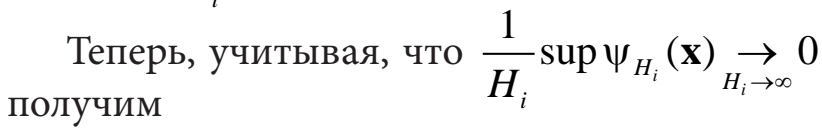

$$
\begin{gathered}
\frac{1}{H_{i}} \int \psi_{H_{i}}^{2}(\mathbf{x}-\mathbf{s}) p\left(\mathbf{s} / \omega_{i}\right) d \mathbf{s} \leq \\
\leq \frac{1}{H_{i}} \sup \psi_{H_{i}}(\mathbf{x}) \int \psi_{H_{i}}(\mathbf{x}-\mathbf{s}) p\left(\mathbf{s} / \omega_{i}\right) d \mathbf{s} \underset{H_{i} \rightarrow \infty}{\rightarrow} 0, \\
\frac{H_{i}-1}{H_{i}}\left(\int \psi_{H_{i}}(\mathbf{x}-\mathbf{s}) p\left(\mathbf{s} / \omega_{i}\right) d \mathbf{s}\right)^{2} \underset{H_{i} \rightarrow \infty}{\rightarrow} p^{2}\left(\mathbf{x} / \omega_{i}\right),
\end{gathered}
$$

и, окончательно

$$
D\left[\tilde{p}\left(\mathbf{x} / \omega_{i}\right)\right]_{H_{i} \rightarrow \infty}^{\rightarrow} 0 .
$$

Утверждение 2. Если плотность распределения $\tilde{p}\left(\mathbf{x} / \omega_{i}\right)$ при $h^{k}\left(H_{i}\right) \underset{H_{i} \rightarrow \infty}{\rightarrow} 0$ и $H_{i} h^{k}\left(H_{i}\right) \underset{H_{i} \rightarrow \infty}{\rightarrow} \infty$ сходится в среднеквадратичном к плотности $p\left(\mathbf{x} / \omega_{i}\right)$ для всех $\mathbf{x}$, то и смешанная оценка $\hat{p}\left(\mathbf{x} / \omega_{i}\right)$ аналогичным образом сходится к $p\left(\mathbf{x} / \omega_{i}\right)$.

Рассмотрим сначала дисперсию стандартной оценки $D\left[\tilde{p}\left(\mathbf{x} / \omega_{i}\right)\right]$ и на основе (8) представим в ней математическое ожидание квадрата оценки в виде

$$
\begin{gathered}
M\left[\tilde{p}^{2}\left(\mathbf{x} / \omega_{i}\right)\right]=\frac{1}{H_{i}} \int \psi_{H_{i}}^{2}(\mathbf{x}-\mathbf{s}) p\left(\mathbf{s} / \omega_{i}\right) d \mathbf{s}+ \\
+\frac{H_{i}-1}{H_{i}}\left(\int \psi_{H_{i}}(\mathbf{x}-\mathbf{s}) p\left(\mathbf{s} / \omega_{i}\right) d \mathbf{s}\right)^{2} .
\end{gathered}
$$

Для первого слагаемого при $\frac{1}{H_{i}} \sup \psi_{H_{i}}(\mathbf{x}) \underset{H_{i} \rightarrow \infty}{\rightarrow} 0$ выполняется

$$
\begin{gathered}
\frac{1}{H_{i}} \int \psi_{H_{i}}^{2}(\mathbf{x}-\breve{\mathbf{s}}-\mathbf{v}) p\left(\breve{\mathbf{s}} / \omega_{i}\right) p(\mathbf{v}) d \breve{\mathbf{s}} d \mathbf{v} \leq \\
\leq \frac{1}{H_{i}} \sup \psi_{H_{i}}(\mathbf{x}) \int \psi_{H_{i}}(\mathbf{x}-\breve{\mathbf{s}}-\mathbf{v}) \times \\
\quad \times p\left(\breve{\mathbf{s}} / \omega_{i}\right) p(\mathbf{v}) d \breve{\mathbf{s}} d \mathbf{v} \underset{H_{i} \rightarrow \infty}{\rightarrow} 0 .
\end{gathered}
$$

Для второго слагаемого в силу сходимости стандартных оценок выполняется

$$
\begin{gathered}
\frac{H_{i}-1}{H_{i}}\left(\int \psi_{H_{i}}(\mathbf{x}-\breve{\mathbf{s}}-\mathbf{v}) p\left(\breve{\mathbf{s}} / \omega_{i}\right) p(\mathbf{v}) d \breve{\mathbf{s}} d \mathbf{v}\right)^{2}= \\
=\frac{H_{i}-1}{H_{i}}\left(\int \vartheta_{H_{i}}(\mathbf{x}-\breve{\mathbf{s}}) p\left(\breve{\mathbf{s}} / \omega_{i}\right) d \breve{\mathbf{s}}\right)^{2} \underset{H_{i} \rightarrow \infty}{\rightarrow} \\
\underset{H_{i} \rightarrow \infty}{\rightarrow} p^{2}\left(\mathbf{x} / \omega_{i}\right) .
\end{gathered}
$$


Теперь рассмотрим дисперсию смешанной оценки

$$
D\left[\hat{p}\left(\mathbf{x} / \omega_{i}\right)\right]=M\left[\hat{p}^{2}\left(\mathbf{x} / \omega_{i}\right)\right]-p^{2}\left(\mathbf{x} / \omega_{i}\right) .
$$

Заметим, сначала, что

$$
\begin{gathered}
\frac{1}{H_{i}} \vartheta_{H_{i}}(\mathbf{x})=\frac{1}{H_{i}} \int \psi_{H_{i}}(\mathbf{x}-\mathbf{v}) p(\mathbf{v}) d \mathbf{v} \leq \\
\leq \frac{1}{H_{i}} \sup \psi_{H_{i}}(\mathbf{x}) \Rightarrow \\
\Rightarrow \frac{1}{H_{i}} \sup \vartheta_{H_{i}}(\mathbf{x}) \leq \frac{1}{H_{i}} \sup \psi_{H_{i}}(\mathbf{x}) .
\end{gathered}
$$

Тогда для смешанной оценки

$$
\begin{gathered}
M\left[\hat{p}^{2}\left(\mathbf{x} / \omega_{i}\right)\right]= \\
=\frac{1}{H_{i}^{2}} \sum_{r=1}^{H_{i}} \sum_{s=1}^{H_{i}} M\left[\vartheta_{H_{i}}\left(\mathbf{x}-\breve{\mathbf{x}}^{(i, r)}\right) \vartheta_{H_{i}}\left(\mathbf{x}-\breve{\mathbf{x}}^{(i, s)}\right)\right]= \\
=\frac{1}{H_{i}^{2}} \sum_{r=1}^{H_{i}} M\left[\vartheta_{H_{i}}^{2}\left(\mathbf{x}-\breve{\mathbf{x}}^{(i, r)}\right)\right]+ \\
+\frac{1}{H_{i}^{2}} \sum_{r=1}^{H_{i}} \sum_{s=1, s \neq r}^{H_{i}} M\left[\vartheta_{H_{i}}\left(\mathbf{x}-\breve{\mathbf{x}}^{(i, r)}\right) \vartheta_{H_{i}}\left(\mathbf{x}-\breve{\mathbf{x}}^{(i, s)}\right)\right]= \\
=\frac{1}{H_{i}} \int \vartheta_{H_{i}}^{2}(\mathbf{x}-\breve{\mathbf{s}}) p\left(\breve{\mathbf{s}} / \omega_{i}\right) d \breve{\mathbf{s}}+ \\
+\frac{H_{i}-1}{H_{i}}\left(\int \vartheta_{H_{i}}(\mathbf{x}-\breve{\mathbf{s}}) p\left(\breve{\mathbf{s}} / \omega_{i}\right) d \breve{\mathbf{s}}\right)^{2} .
\end{gathered}
$$

1 При этом первое слагаемое при $\frac{1}{H_{i}} \sup \psi_{H_{i}}(\mathbf{x}) \underset{H_{i} \rightarrow \infty}{\rightarrow} 0$ на основе (10) стремится к нулю, так как

$$
\begin{gathered}
\frac{1}{H_{i}} \int \vartheta_{H_{i}}^{2}(\mathbf{x}-\breve{\mathbf{s}}) p\left(\breve{\mathbf{s}} / \omega_{i}\right) d \breve{\mathbf{s}} \leq \\
\leq \frac{1}{H_{i}} \sup \vartheta_{H_{i}}(\mathbf{x}) \int \vartheta_{H_{i}}(\mathbf{x}-\breve{\mathbf{s}}) p\left(\breve{\mathbf{s}} / \omega_{i}\right) d \breve{\mathbf{s}} \underset{H_{i} \rightarrow \infty}{\rightarrow} 0,
\end{gathered}
$$

а для второго слагаемого выполняется (9), т. е. математические ожидания квадрата стандартной и смешанной оценок стремятся к одному пределу, определяемому величиной $p^{2}\left(\mathbf{x} / \omega_{i}\right)$. Таким образом, $D\left[\hat{p}\left(\mathbf{x} / \omega_{i}\right)\right]_{H_{i} \rightarrow \infty}^{\rightarrow} 0$, что позволяет говорить о том, что смешанная оценка является состоятельной.

Утверждение 3. При известной плотности распределения $p(\mathbf{v}), M[\mathbf{v}]=0$ и фиксированной выборке $\breve{X}^{H_{i}}=\left\{\breve{\mathbf{x}}^{(i, 1)}, \ldots, \breve{\mathbf{x}}^{\left(i, H_{i}\right)}\right\}$ использование смешанной оценки эквивалентно реализации процедуры искусственного размножения обучающих данных, в ходе которой для каждого вектора $\breve{\mathbf{x}}^{(i, k)} \in \breve{X}^{H_{i}}, k=\overline{1, H_{i}}$ проводится генерация подвыборки, состоящей из $P_{i, k} \rightarrow \infty$ образов, на основе распределения $p\left(\mathbf{w} /\left\{\breve{\mathbf{x}}^{(k)}, \omega_{i}\right\}\right)$, где $\mathbf{w}^{(i)}=\breve{\mathbf{x}}^{(i, k)}+\mathbf{v}\left(\breve{\mathbf{x}}^{(i, k)}\right.$ выступает в роли математического ожидания для распределения подвыборки).

Доказательство вытекает из сходимости ядерной оценки $\hat{p}\left(\mathbf{x} /\left\{\breve{\mathbf{x}}^{(k)}, \omega_{i}\right\}\right)$, восстановленной по подвыборке $X^{\left(P_{i, k}\right)}=\left\{\mathbf{x}^{(i, k, 1)}, \ldots, \mathbf{x}^{\left(i, k, P_{i, k}\right)}\right\}$, к плотности $p\left(\mathbf{w} /\left\{\breve{\mathbf{x}}^{(k)}, \omega_{i}\right\}\right)$ при $P_{i, k} \rightarrow \infty$. Для генерации подвыборки в этом случае может быть использован метод нелинейного функционального преобразования или метод исключений с соответствующей коррекцией вида плотности $p(\mathbf{v})$ для учета усеченного характера плотности генерируемых данных.

\section{3. РЕЗУЛЬТАТЫ ЭКСПЕРИМЕНТАЛЬНЫХ ИССЛЕДОВАНИЙ}

Исследование различных вариантов алгоритмов распознавания изображений проводилось в среде MATLAB методом статистического моделирования.

Первоначально в ходе экспериментальных исследований производился анализ алгоритмов распознавания искусственно синтезированных изображений, подверженных ДИ и аддитивным помехам. Использовались изображения двух видов: изображения колокольной (гауссовской) функции с различной степенью вытянутости и ориентации (рис. 1, на графиках далее обозначены как gaussian) и изображения, полученные на основе двумерного гармонического ряда (рис. 2, на графиках далее обозначены как chess). Исходные размеры изображений составляли $21 \times 21$ пиксель.

В ходе сравнительного исследования алгоритмов распознавания изображений рассматривались следующие варианты их построения:

- алгоритм на основе смешанных непараметрических ядерных оценок $\hat{p}\left(\mathbf{x} / \omega_{i}\right), i=1,2$ (5);

- алгоритм на основе стандартных непараметрических ядерных оценок $\tilde{p}\left(\mathbf{x} / \omega_{i}\right)$, $i=\overline{1,2}$;

- алгоритм на основе гауссовского приближения функций правдоподобия 


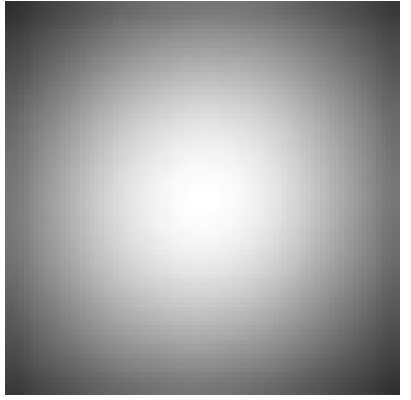

$a$

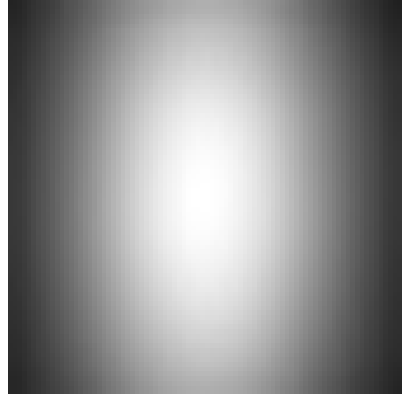

6

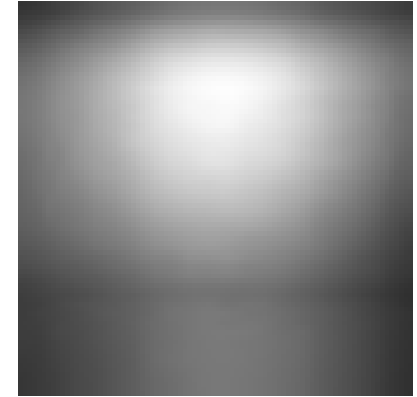

B

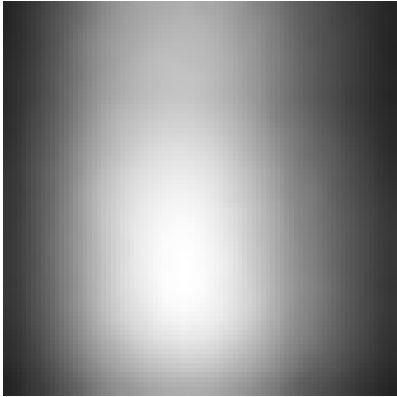

2

Рис. 1. Синтезированные изображения $(a, 6)$ в виде колокольной (гауссовской) функиии и примеры их дебормаций (в, г), в на основе а и г на основе 6

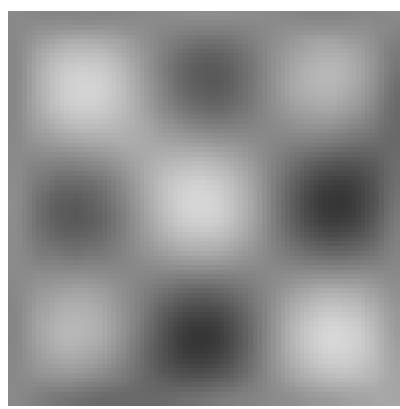

$a$

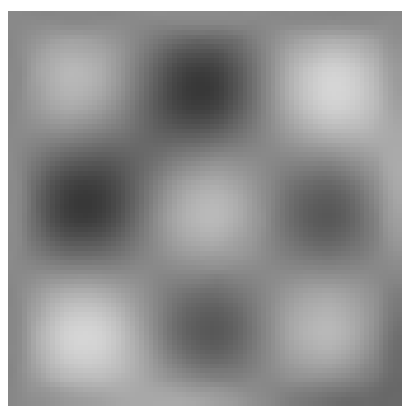

$\sigma$

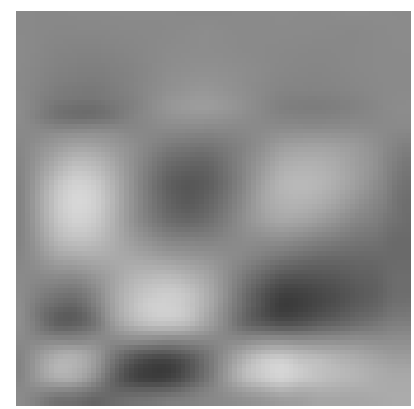

B

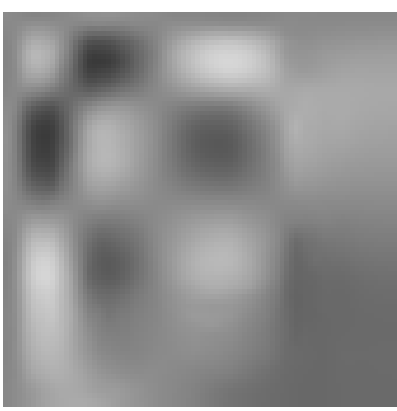

2

Рис. 2. Синтезированные изображения ( $(, 6)$, полученные на основе двумерного гармонического ряда, и примеры их дебормаций $(8,2)$, в на основе а и г на основе $б$

$\widehat{p}\left(\mathbf{x} / \omega_{i}\right)=N\left(\mathbf{x}, \mathbf{m}_{i}, \mathbf{C}_{i}\right), \quad i=\overline{1,2}$ с использованием оценок математических ожиданий и матриц ковариаций классов $\mathbf{m}_{i}, \mathbf{C}_{i}, i=\overline{1,2}$;

- алгоритм на основе искусственной двуслойной нейронной сети прямого распространения с $2 K$ нейронами в скрытом слое, обучаемой по выборкам $X^{H_{i}}=\left\{\mathbf{x}^{(i, 1)}, \ldots, \mathbf{x}^{\left(i, H_{i}\right)}\right\}$, $i=\overline{1,2}$, где $K$ соответствует числу главных компонент в (2).

В плане сравнительного анализа последний алгоритм представляет особый интерес, поскольку, в отличие от использования смешанной ядерной оценки или классической параметрической оценки, в стандартном варианте его реализации отсутствует возможность учета аддитивного шума (нейросети можно обучать только по исходным выборкам). Поэтому для учета влияния аддитивного шума в рассматриваемой задаче целесообразно дополнительно проводить искусственное размножение данных.

Корреляционные функции вносимых ДИ и аддитивного шума в ходе экспериментов задавались в виде

$$
\begin{gathered}
\mathrm{B}_{0}\left(x-x^{\prime}, y-y^{\prime}\right)= \\
=A_{m}^{2} \exp \left(-\alpha_{0}\left(\left|x-x^{\prime}\right|+\left|y-y^{\prime}\right|\right)\right), \\
\sigma^{2} \mathrm{R}\left(x-x^{\prime}, y-y^{\prime}\right)= \\
=\sigma^{2} \exp \left(-\alpha_{\mathrm{v}}\left(\left|x-x^{\prime}\right|+\left|y-y^{\prime}\right|\right)\right),
\end{gathered}
$$

где параметры $\alpha_{0}, \alpha_{\mathrm{v}}$ определялись исходя из задаваемых значений коэффициентов корреляции $r_{0}$ и $r_{\mathrm{v}}$ соседних элементов случайных полей. Для всех представленных графиков использовались следующие значения параметров функции деформации: $A_{m}=0,25$; $r_{0}=0,995$.

Результаты, полученные при распознавании синтезированных изображений (рис. 1, 2), представлены на рис. 3, 4 в виде зависимостей вероятности суммарной ошибки распознавания $E_{r}=p\left(\omega_{1}\right) \alpha+p\left(\omega_{2}\right) \beta$ от величины отношения сигнал-шум, которое задавалось как отношение пикового значения яркости изображений к $\sigma$

$$
\rho_{r}=\frac{\max \left(\mathbf{s}_{1}, \mathbf{s}_{2}\right)}{\sigma} .
$$


Модели и алгоритмы распознавания цифрровых изображений ...

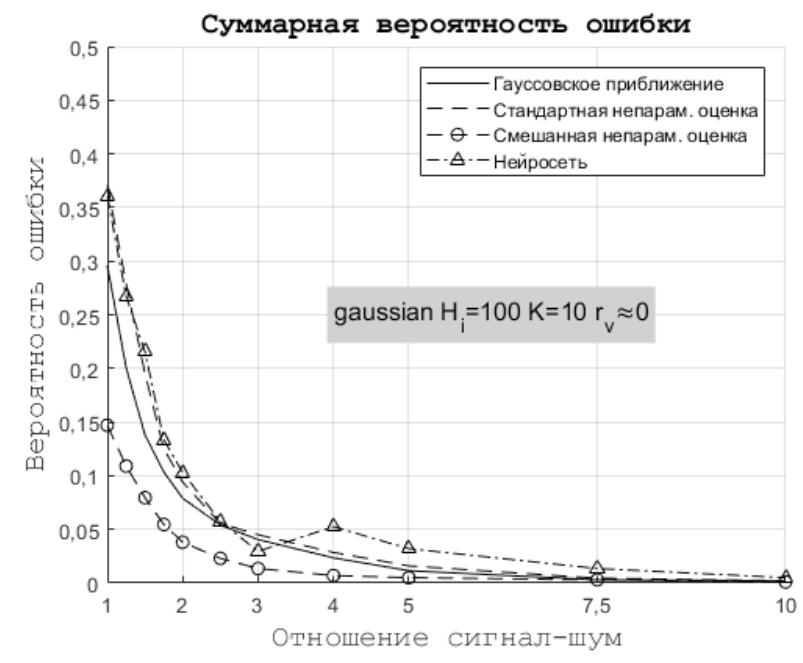

$a$

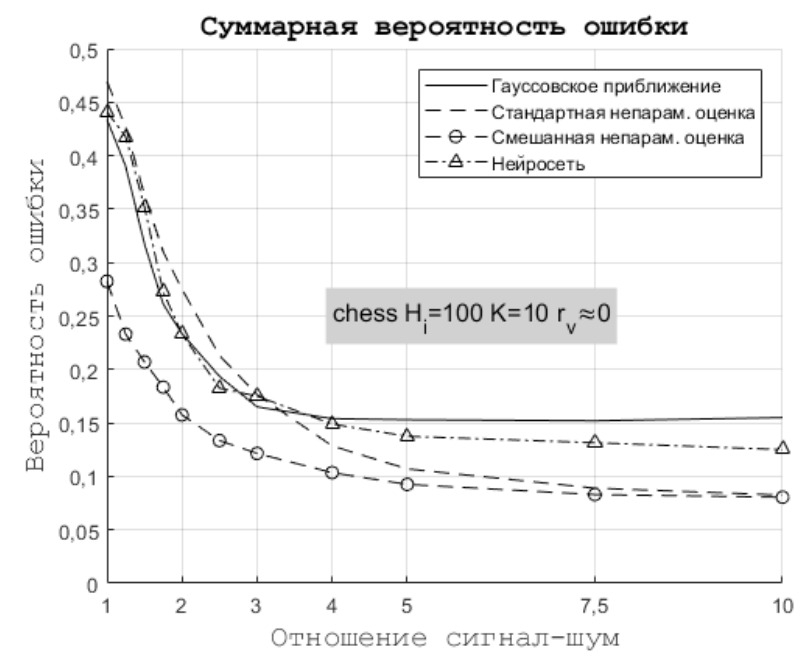

B

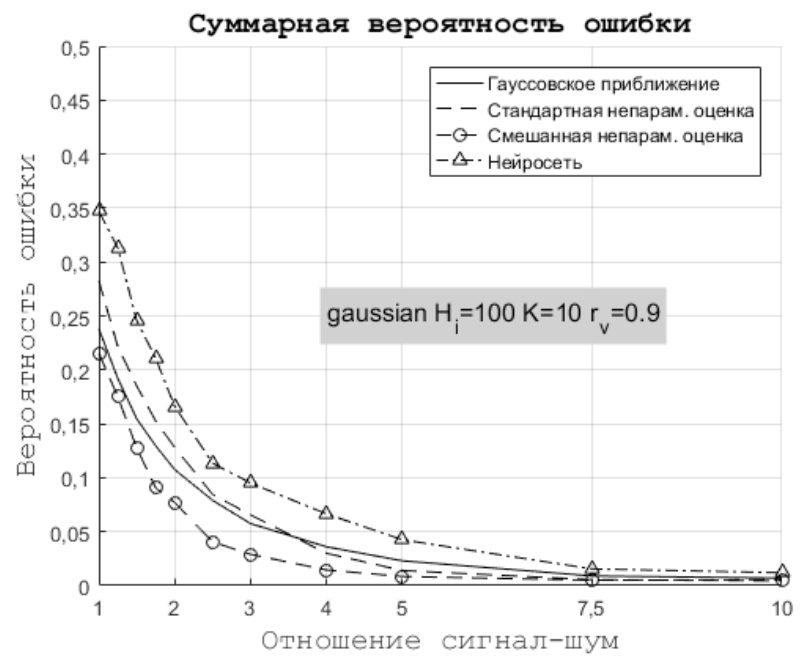

6

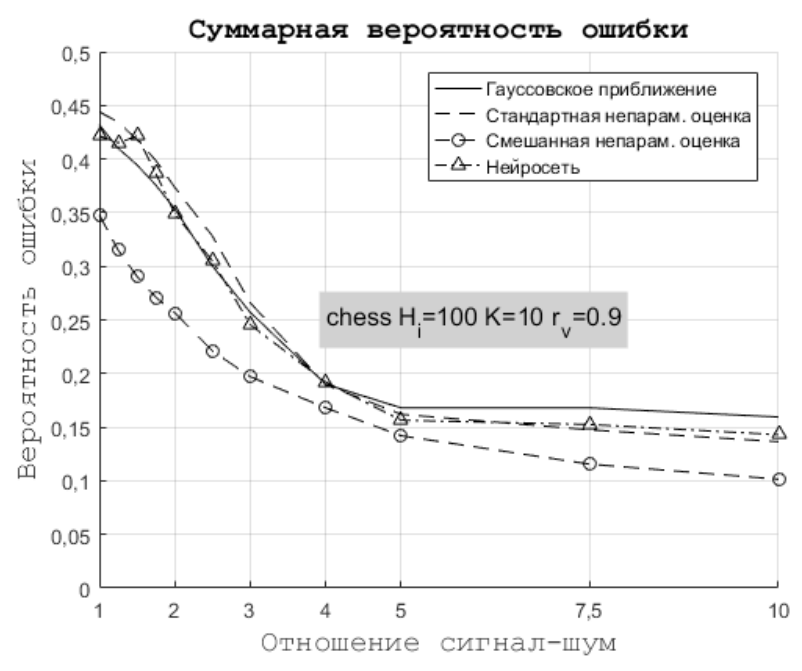

2

Рис. 3. Зависимости для оценок вероятности ошибки распознавания синтезированных изображений (рис. 1, 2) от отношения сигнал-шум для $H_{i}=100$ и $\mathrm{K}=10$

На рис. 3, 4 представлены результаты, полученные на основе имитационного моделирования алгоритмов распознавания синтезированных изображений с усреднением по 10 реализациям их работы при $K=10$ : на рис. 3 для $H_{i}=100$, и на рис. 4 - для $H_{i}=500$. Здесь и далее обозначения на графиках, вынесенные в текстовую дорожку, соответствуют вариантам задания исходных данных: gaussian, chess - тип распознаваемых изображений; $H_{i}$ - число элементов обучающей выборки; $K$ соответствует количеству главных компонент; $r_{\mathbf{v}}$ - коэффициент корреляции соседних элементов аддитивного шума. Представленные ниже зависимости получены для двух ва- риантов: воздействие аддитивного белого шума с низким уровнем корреляции $r_{\mathrm{v}} \approx 0$ (рис. 3,4 , а, в) и воздействие значительно коррелированного аддитивного шума с коэффициентом $r_{\mathrm{v}}=0,9$ (рис. $3,4,6$, г).

На втором этапе наших исследований были осуществлены эксперименты по распознаванию реальных изображений элементов зерновых смесей (ЭЗС, на графиках далее обозначены как seeds) пшеницы (рис. 5, а) и ячменя (рис. 5, б), полученных при съемке движущегося неоднородного потока семян в системе фотосепарации реального времени [6]. Индивидуальные отличия формы ЭЗС каждого класса при этом трактовались как 


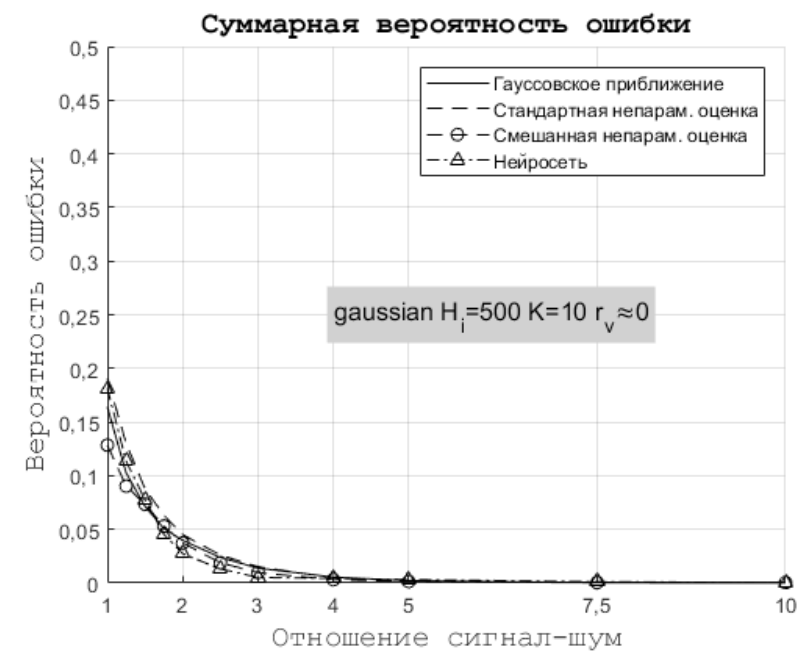

$a$

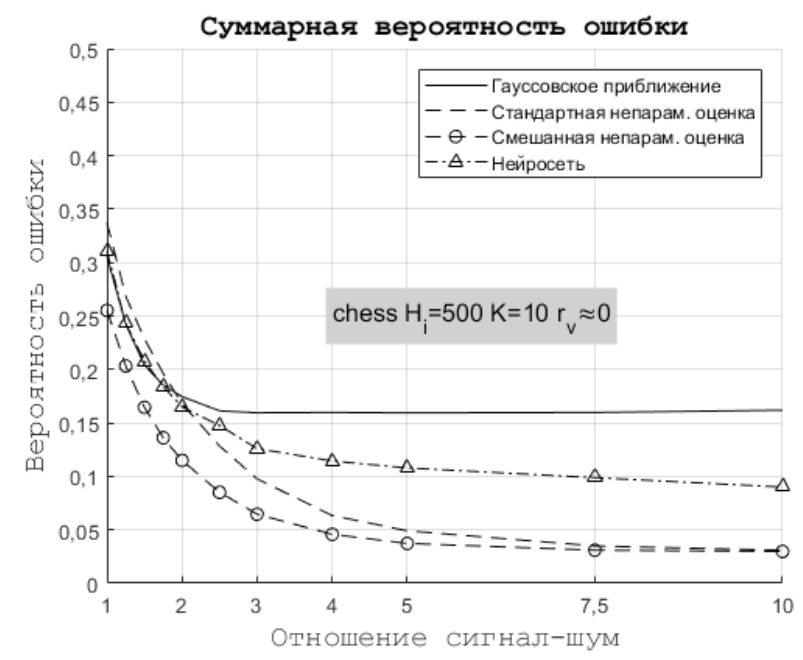

B

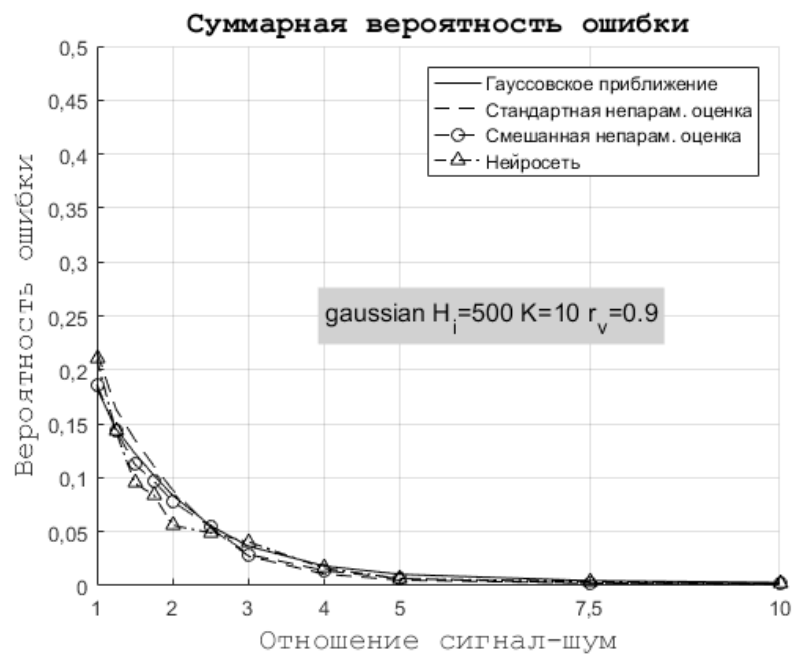

6

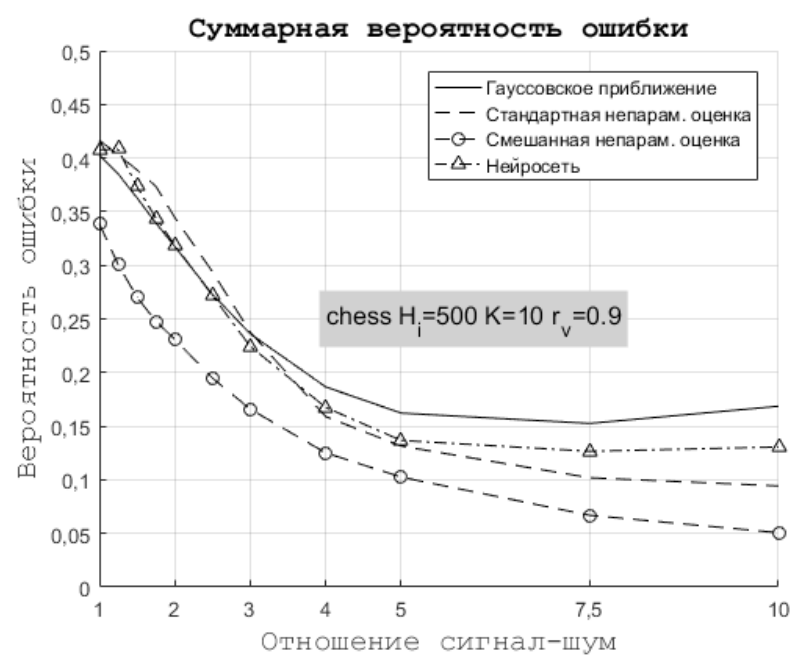

2

Рис. 4. Зависимости для оценок вероятности ошибки распознавания синтезированных изображений (рис. 1, 2) от отношения сигнал-шум для $H_{i}=500$ и $\mathrm{K}=10$

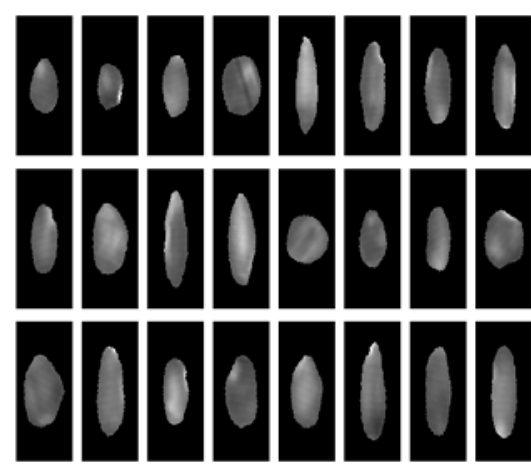

$a$

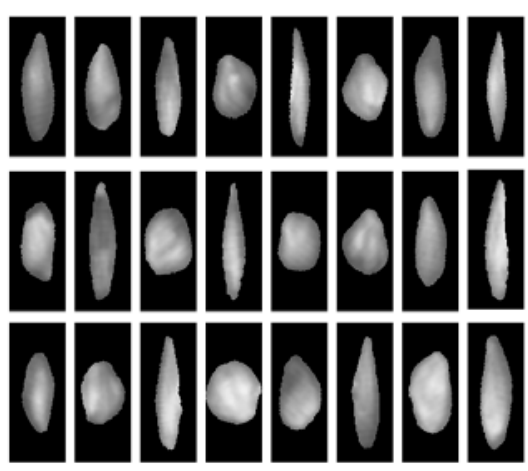

6

Рис. 5. Примеры подготовленных реальных изображений элементов зерновых смесей пшеницы (а) и ячменя (б), использованньх при обучении алгоритмов распознавания 
Модели и алгоритмы распознавания цифрровых изображений ...

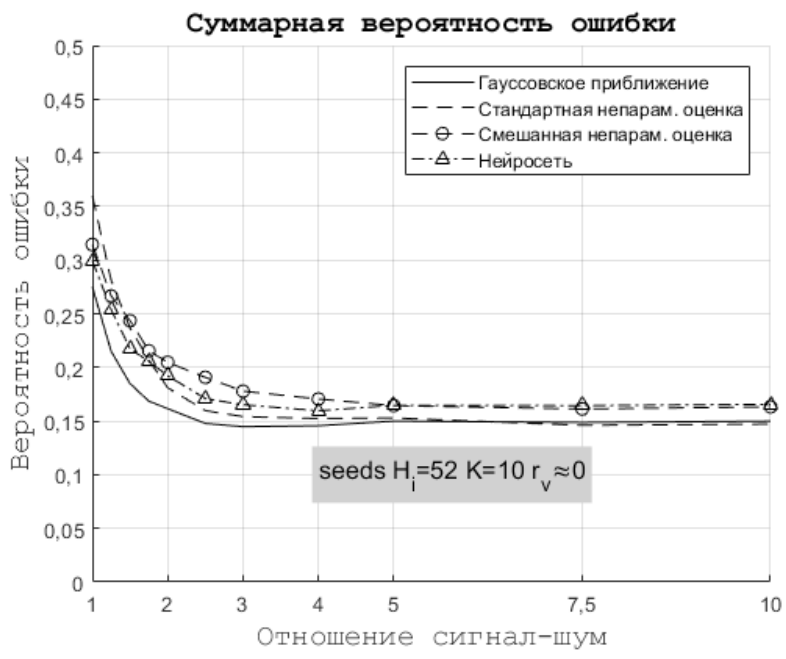

$a$

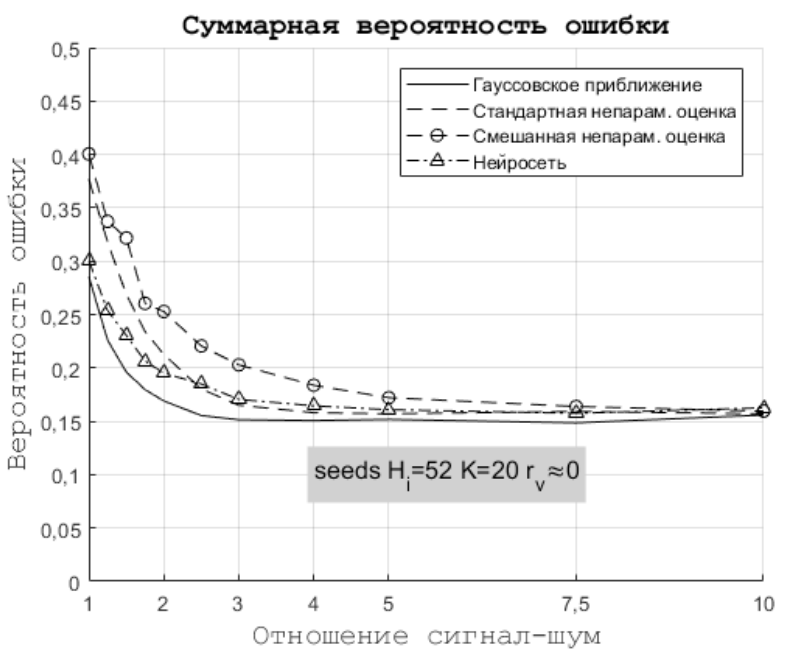

B

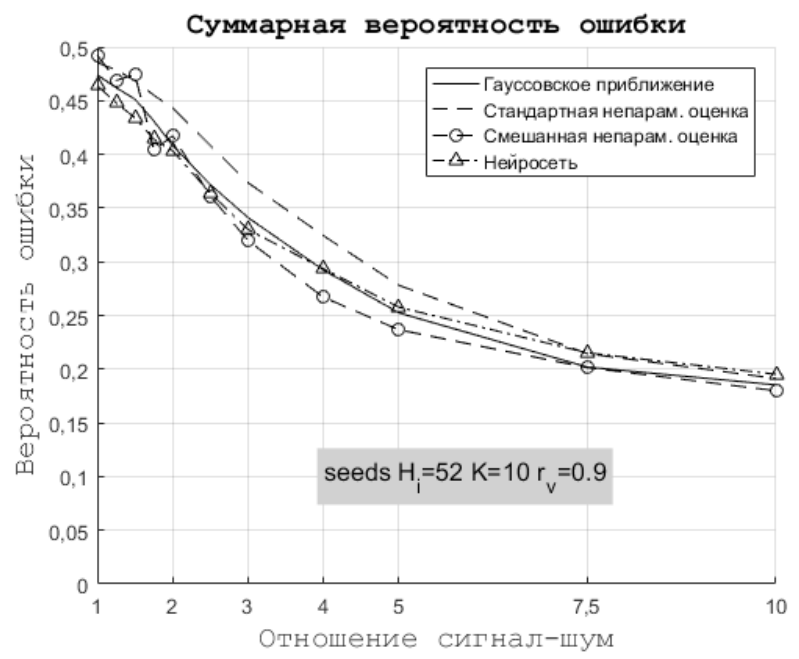

6

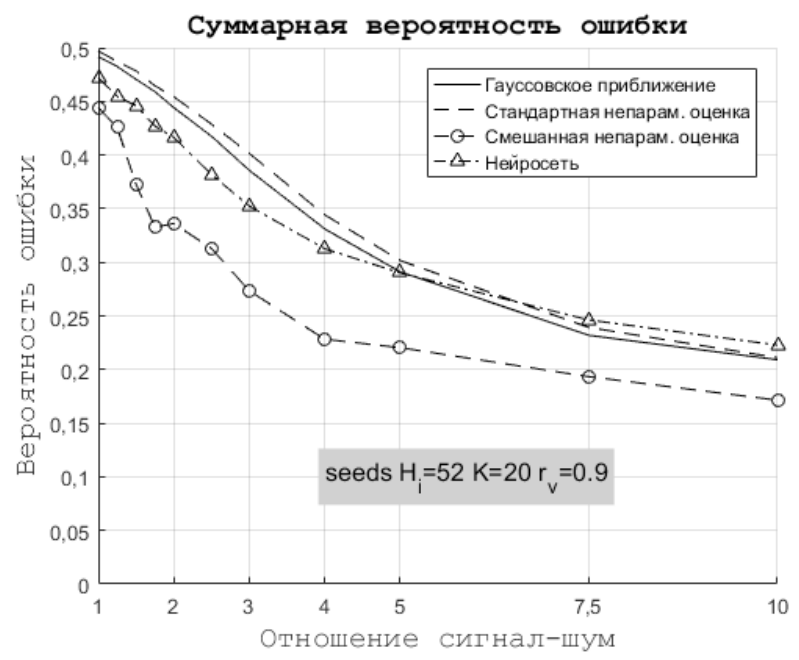

2

Рис. 6. Зависимости для оценок вероятности ошибки распознавания изображений элементов зерновых смесей (рис. 5) от отношения сигнал-иум при $H_{i}=52$ и $K=\{10,20\}$

деформирующие искажения. Зависимости для оценок вероятности ошибки распознавания этих изображений, полученные при значениях $r_{\mathrm{v}} \approx 0$ и $r_{\mathrm{v}}=0,9$ для $K=\{10,20\}$ и $H_{i}=52$ с усреднением по 100 реализациям работы алгоритмов, представлены на рис. 6.

\section{4. ОБСУЖДЕНИЕ РЕЗУЛЬТАТОВ}

При оценке вероятности ошибки распознавания изображений, имеющих вид колокольной (гауссовской) функции (рис. 1), при малой величине обучающей выборки характерно преимущество алгоритмов, основанных на параметрических и непараметриче- ских оценках, по отношению к алгоритму, основанному на использовании нейронной сети (рис. 3, а, 6 и рис. 4, а, б). Это преимущество растет с увеличением коэффициента корреляции аддитивного шума, что, на наш взгляд, связано с однородностью структуры подвергаемых ДИ изображений и превалирующим в этом случае влиянием аддитивных помех. Это приводит к тому, что законы распределения значений главных компонент распознаваемых изображений достаточно хорошо описываются в гауссовском приближении, на котором и основаны использованные оценки. При малом объеме обучающей выборки выявлено значительное превосход- 


\section{А. В. Акимов, А. О. Донских, А. А. Сирота}

ство смешанной непараметрической оценки перед всеми другими алгоритмами, которое нивелируется с увеличением объема обучающих данных, что согласуется с представленными выше теоретическими выкладками.

Для полученных на основе двумерного гармонического ряда исходных изображений квазипериодической структуры (рис. 2), которая сильно подвержена влиянию со стороны ДИ, характерно еще более выраженное, чем в предыдущем случае, превосходство смешанной непараметрической оценки перед всеми другими алгоритмами, которое незначительно уменьшается при увеличении объема обучающей выборки со 100 до 500 экземпляров (рис. 3, в, г и рис. 4, в, г). Нейросетевой алгоритм здесь в большинстве случаев показывает себя наравне с алгоритмами, основанными на параметрической и стандартной непараметрической оценках. Для последней, однако, вместе с увеличением отношения сигнал-шум характерны постепенное приближение к значениям, соответствующим смешанной непараметрической оценке, и отклонение от результатов работы остальных двух алгоритмов.

При распознавании изображений ЭЗС (рис. 6), подвергнутых дополнительному воздействию аддитивного шума, была получена несколько иная картина. Для некоррелированного шума (левый столбец на рис. 6), лучшие результаты показывает алгоритм, основанный на гауссовском приближении с использованием параметрических оценок. Это, по нашему мнению, связано с малым объемом доступных обучающих данных $\left(H_{i}=52\right)$ и близостью закона распределения значений главных компонент, полученных при преобразовании относительно однородных изображений ЭЗС (рис. 5), к гауссовскому закону распределения. Однако в композиции с коррелированным аддитивным шумом влияние последнего на закон распределения этих значений оказывается столь значительным, что учет параметров аддитивного шума в смешанной оценке позволяет получить наиболее точные результаты (рис. 6 , в, г). С увеличением числа главных компонент данный эффект становится все более выраженным, в то время как точность работы всех остальных алгоритмов снижается.

\section{ЗАКЛЮЧЕНИЕ И БЛАГОДАРНОСТИ}

На основе полученных результатов можно сделать выводы о применимости предложенных моделей и синтезированных алгоритмов распознавания цифровых изображений в условиях деформирующих искажений и аддитивных помех. Показано, что использование алгоритмов, основанных на смешанных ядерных оценках, эквивалентно реализации процедуры искусственного размножения обучающих данных в соответствии со статистической моделью дополнительных аддитивных искажений, оказывающих влияние на распознаваемые изображения, что представляет собой два возможных пути улучшения точности работы алгоритмов распознавания в условиях малых объемов обучающих выборок. Искусственное размножение обучающих данных при этом становится единственной альтернативой при построении обучаемых алгоритмов распознавания, в которых невозможно напрямую учесть параметры используемых статистических моделей данных, например, алгоритмов, основанных на использовании нейронных сетей. В случае недостаточного объема обучающих данных выигрыш за счет использования смешанных оценок можно получить в условиях, когда искажения, эффекты которых требуется учесть, оказывают существенное влияние на законы распределения используемых признаков, например, в случае наличия коррелированного аддитивного шума.

Работа выполнена в рамках госзадания Минобрнауки РФ по проекту № 8.3844.2017/4.6 "Разработка средств экспресс-анализа и классибикации элементов неоднородного потока зерновых смесей с патологиями на основе интеграции методов спектрального анализа и машинного обучения».

\section{СПИСОК ЛИТЕРАТУРЫ}

1. Begum, N. Accelerating Dynamic Time Warping Clustering with a Novel Admissible Pruning Strategy / N. Begum, L. Ulanova, J. Wang, E. Keogh // Proceedings of the 21th ACM SIGKDD International Conference on Knowledge Discovery and Data Mining. - 2015. - V. 7(3). P. 49-58. - DOI: 10.1145/2783258.2783286. 
2. Aghabozorgi, S. Time-series Clustering A Decade Review / S. Aghabozorgi, A. S. Shirkhorshidi, T. Ying Wah. - Information Systems. - 2015. - V. 53. - P. 16-38. - DOI: 10.1016/ j.is.2015.04.007.

3. Rabiner, L. Fundamentals of Speech Recognition / L. Rabiner, B. H. Juang. - Englewood Cliffs, NJ: Prentice Hall PTR, 1993. - 507 p.

4. Corradini, A. Dynamic time warping for offline recognition of a small gesture vocabulary / A. Corradini. // Proceedings of the IEEE ICCV Workshop on Recognition, Analysis, and Tracking of Faces and Gestures in Real-Time Systems. - 2001. - P. 82-89. - DOI: 10.1.1.200.2035.

5. Vemulapalli, R. Human Action Recognition by Representing 3D Skeletons as Points in a Lie Group / R. Vemulapalli, F. Arrate, R. Chellappa // IEEE Conference on Computer Vision and Pattern Recognition, June 23-28, 2014 (CVPR 2014). - 2014. - P. 588-595. - DOI: 10.1109/ CVPR.2014.82.

6. Алгазинов, Э. К. Аппаратно-программный комплекс для анализа неоднородного потока объектов в системах фотосепарации реального времени / Э. К. Алгазинов, М. А. Дрюченко, А. А. Сирота, Д. А. Минаков, В. А. Шульгин // Измерительная техника. 2014. - № 5. - C. 23-29.

7. Keysers, D. Deformation models for image recognition / D. Keysers, T. Deselaers, C. Gollan, H. Ney // IEEE Transactions on Pattern Analysis and Machine Intelligence. - 2007. - V. 29(8). P. 1422-1435. - DOI: 10.1109/TPAMI.2007.1153.

8. Felzenswalb, $P$. A discriminatively trained, multiscale, deformable partmodel/P. Felzenswalb, D. McAllester, D. Ramanan // IEEE Conference on Computer Vision and Pattern Recognition, June 24-26, 2008 (CVPR 2008). - 2008. - P. 1-8. DOI: 10.1109/CVPR.2008.4587597.

9. Drap, P. An Exact Formula for Calculating Inverse Radial Lens Distortions / P. Drap, J. Lefèvre // Sensors. - 2016. - V. 16(6). P. 1-18. - DOI: 10.3390/s16060807.

10. Friston, K. J. Statistical parametric mapping: the analysis of functional brain images / K. J. Friston, W. D. Penny, J. Ashburner, S. J. Kiebel, T. E. Nichols. - London: Academic Press, 2006. -656 p.
11. Brown, L. G. A Survey of Image Registration Techniques / L.G. Brown // ACM Computing Surveys. - 1992. - V. 24(4). - P. 325-376. DOI: $10.1145 / 146370.146374$.

12. Fortun, D. Optical flow modeling and computation: A survey / D. Fortun, P. Bouthemy, C. Kervrann // Computer Vision and Image Understanding, Elsevier. - 2015. - V. 134. P. 1-21. - DOI: 10.1016/j.cviu.2015.02.008.

13. Zope, B. A Survey of Morphing Techniques / B. Zope, S.B. Zope // International Journal of Advanced Engineering, Management and Science (IJAEMS) - 2017. - V. 3(2). - P. 81-87. DOI: 10.24001/ijaems.3.2.15.

14. Liao, J. Automating Image Morphing Using Structural Similarity on a Halfway Domain / J. Liao. R.S. Lima, D. Nehab, H. Hoppe, P.V. Sander, J.Yu // ACM Transactions on Graphics (TOG). - 2014. - V. 33(5). - P. 1-12. DOI: $10.1145 / 2629494$.

15. Акимов, А. В. Модели и алгоритмы искусственного размножения данных для обучения алгоритмов распознавания лиц методом Виолы-Джонса / А. В. Акимов, А. А. Сирота // Компьютерная оптика. - 2016. - Т. 40, № 6. - C. 911-918. - DOI: 10.18287/2412-61792016-40-6-911-918.

16. Небаба, С. Г. Алгоритм построения деформируемых 3D моделей лица и обоснование его применимости в системах распознавания личности / С. Г. Небаба, А. А. Захарова // Труды СПИИРАН. - 2017. - Вып. 52. - С. 157179. - DOI: 10.15622/sp.52.8.

17. Акимов, А. В. Синтез и анализ алгоритмов распознавания цифровых сигналов в условиях деформирующих искажений и аддитивных помех / А. В. Акимов, А. А. Сирота // Известия вузов. Радиотехника. - 2017. T. 60, № 10. - C. 592-604. - DOI: 10.20535/ S0021347017100041.

18. Fukunaga, K. Introduction to statistical pattern recognition / K. Fukunaga. - San Diego, CA: Academic Press, 1990. - 591 p.

19. Сupoma, A. A. Методы и алгоритмы анализа данных и их моделирование в MATLAB / А.А. Сирота. - СПб.: БХВ-Петербург, 2016. - 384 c.

20. Кривенко, М. П. Непараметрическое оценивание элементов байесовского класси- 
фикатора / М. П. Кривенко // Информатика и ее применения. - 2010. - Т. 4, № 2. - С. 13-24.

21. Duda, R. O. Pattern Classification / R. O. Duda, P. E. Hart, D. G. Stork. - Second Edition. - Hoboken, NJ: Wiley-Interscience, 2000. $-680 \mathrm{p}$.

22. Абезгауз, Г. Г. Справочник по вероятностным расчетам / Г. Г. Абезгауз, А. П. Тронь,

Акимов А. В. - исследователь, преподаватель-исследователь, младший научный сотрудник кафедры технологий обработки и защиты информации, факультет компьютерных наук, Воронежский государственный университет. E-mail: akimov@vsu.ru

Донских А. О. - аспирант кафедры технологий обработки и защиты информации, факультет компьютерных наук, Воронежский государственный университет.

E-mail: a.donskikh@outlook.com

Сирота А. А. - д. т. н., профессор, заведующий кафедрой технологий обработки и защиты информации, факультет компьютерных наук, Воронежский государственный университет. E-mail: sir@cs.vsu.ru
Ю. Н. Копенкин, И. А. Коровина. - Издание второе. - М. : Воениздат, 1970. - 536 с.

23. Крянев, А. В. Математические методы обработки неопределенных данных / А. В. Крянев, Г. В. Лукин. - М. : Физмалит, 2003. - 216 с.

Akimov A. V. - Researcher, Teacher-researcher, Junior Researcher of Department of Processing Technology and Information Security, Computer Sciences Faculty, Voronezh State University.

E-mail: akimov@vsu.ru

Donskikh A. O. - Postgraduate Student, Department of Processing Technology and Information Security, Computer Sciences Faculty, Voronezh State University.

E-mail: a.donskikh@outlook.com

Sirota A. A. - Doctor of Technical Sciencies, Professor, Head of Department of Processing Technology and Information Security, Computer Sciences Faculty, Voronezh State University. E-mail: sir@cs.vsu.ru 